\title{
EXPLORING CULTURAL HERITAGE COLLECTIONS IN IMMERSIVE ANALYTICS: CHALLENGES, BENEFITS, AND A CASE STUDY USING VIRTUAL REALITY
}

\author{
Kathrin Koebel $^{1 *}$, Doris Agotai ${ }^{1}$, Arzu Çöltekin ${ }^{1}$ \\ ${ }^{1}$ University of Applied Sciences and Arts Northwestern Switzerland FHNW, Institute for Interactive Technologies IIT, \\ Brugg-Windisch, Switzerland - (kathrin.koebel, doris.agotai, arzu.coltekin)@fhnw.ch
}

Commission IV, WG IV/9

KEY WORDS: Virtual Reality, Immersive Analytics, Visual Analytics, Data Exploration, 3D User Interfaces, Interaction Design, User Experience, Cultural Heritage, Interactive Archives and Collections

\begin{abstract}
:
Immersive analytics, at the intersection of visual analytics and virtual reality has recently gained some traction. Taking a similar approach, VaRt-DataExplorer project is concerned with exploration of data spaces in Virtual Reality (VR) in the context of cultural heritage collections. Our main objective is to facilitate better a understanding and insight into spatially referenced cultural heritage data sets. Within the scope of the project, this goal would be achieved by providing potentially 'intuitive' forms of real time interaction with the data, and rendering quickly recognizable visuospatial representations to offer more context to cultural artifacts. In particular, a spatial context is provided to the viewers by referencing geographical aspects of the data. Due to the incomplete and imprecise nature of data in this domain, thoughtful attention is given to visualization fidelity. Our initial user study suggests that using an immersive VR offers benefits for the exploration task for the viewer and the user experience provided by VaRt-DataExplorer has received high ratings.
\end{abstract}

\section{INTRODUCTION}

Museums and archives possess a significant amount of cultural heritage data with spatial coverage that often remains unused due to a variety of challenges including issues such as incomplete or uncertain data, or access to technical expertise. Real value can be created for our global cultural understanding if this information is processed and visualized in a meaningful way and made accessible for exploration and discovery. The VaRtDataExplorer project (whereby VaRt is a word play on VR and Art) presented in this paper is an attempt to contribute towards filling this gap, and with this goal in mind, the use of Virtual Reality (VR) for exploring such cultural heritage content is investigated. In this exploration, we document the lessons learned using both quantitative and qualitative methods. In this paper, we describe an applied research project based on the dataset of the online collection of Museum Rietberg Zurich. Museum Rietberg is an art museum that focuses on non-European cultures. The museum features internationally renowned permanent and temporary collections of art from a large geographical coverage including various regions of Asia, Africa and Ancient America. In addition to sculptures, ceramics, paintings, historical photographs, textiles and other physical objects on display in the exhibition, the museum has an even more extensive collection. Public access to the archive of this comprehensive collection is offered via a website (Museum Rietberg, 2020) and the audience thereof consists of people with interest in culture and arts, students and researchers as well as professionals such as art historians and curators. Transforming this ordinary online collection into a virtual interactive exploration space offers an interesting use case for this research, which we are targeting to the general audience (i.e., non-expert users).

We believe our key findings in this project will be relevant to other types of archives or collections in the domain of cultural

\footnotetext{
* Corresponding author
}

heritage, as well as to other domains that work with geographically referenced datasets. The goal of this research is to facilitate exploring a 'data space' in VR by the means of interaction with, and visualization of, geographically referenced cultural heritage data. In contrast with traditional 2D representations, $3 \mathrm{D}$ visualization in immersive VR environments afford novel forms of spatial interaction with data. These novel spatial interaction possibilities might be troubling (and indeed are still subject to research (Stephanidis et al., 2019)) but they may also be rewarding as humans use their hands for countless everyday tasks (Napier et al., 1993). Modern VR devices do offer relatively precise hand-tracking, and as a result of their directness in manipulation, which is reducing the gulf of execution (Hutchins et al., 1985), unlocking some potentially 'intuitive' interaction possibilities (Sherman, Craig, 2018), even for users with no or little previous experience with latest technologies (Blackler et al., 2010). Besides the possibilities that VR might offer, simply by devising strategies for filtering, search and selection of objects as well as developing suitable forms of visualization with reference to the geographical, chronological and contextual aspects enable users to identify patterns, correlations and dependencies among entries. We believe these spatial interaction techniques and contextualized 3D data visualization will open new perspectives that lead to deeper understanding of multi-dimensional contents of datasets and fresh insights; and the participants will find the exploration process to be an engaging, enjoyable and memorable experience.

\section{THEORY AND RELATED WORK}

\subsection{Benefits of VR for Data Exploration}

According to Idreos (2015) data exploration is defined as "efficiently extracting knowledge from data even if we do not know exactly what we are looking for". To achieve this objective advanced data visualization and alternative exploration interfaces 
that help users navigate the underlying data space are important (Idreos et al., 2015). Even though benefits of using 3D in visualization for 'anything' is heavily debated among visualization professionals (Çöltekin et al., 2016, Shepherd, 2008, Schnürer et al., 2020), several researchers have shown that stereoscopic depth perception and VR can benefit data visualization and exploration $e . g$., for helping people better understand their data while exploiting the strengths of human perceptual abilities. McIntre et al. (2014) demonstrate that using stereoscopic visualization improves people's performance in spatial tasks in more than $60 \%$ of the time (McIntire et al., 2014). With the use of appropriate depth cues, 3D visualization can improve the intelligibility of the data, and allow disambiguation of complex abstract representations (Cliquet et al., 2017). In fact, since their inception, virtual environments such as CAVEs have been used for immersive data visualization and analytics and the rise of inexpensive stereoscopic head mounted displays increased the use of VR for this purpose (Wagner Filho et al., 2018). LaViola et al. (2009) state that "there is a mounting evidence that immersive Virtual Reality's ability to let users be 'inside' their data or model and interact directly with the data through body-centric interaction (e.g., by moving their heads, bodies and hands) speeds up the processes of perception and interpretation" (LaViola et al., 2009, p. 226). Such direct manipulation interactions offered by immersive VR environments can be more fluid and more efficient in comparison to non-immersive data displays such as the standard desktop environment with interaction via keyboard and mouse-driven GUI, and thus reduce the cognitive load of the user (LaViola et al., 2009). Ongoing research in immersive analytics investigates how new interaction and display technologies can be used to create more engaging and immersive experiences and seamless workflows and thus support analytical reasoning and decision making. In this context, bringing attention to higher-level usability and design issues in creating effective user interfaces for data analytics in immersive environments appears to be an important research area (Chandler et al., 2015, Çöltekin et al., 2019a).

\subsection{Visual Information Seeking Mantra}

A pivotal theory in data visualization and exploration literature is Shneiderman's Visual Information Seeking Mantra “overview first, zoom and filter, then details on demand" (Shneiderman, 1996). In the case of a geographic visualization, overview could be an aerial (bird's eye view) perspective for broader spatial context, and zooming in on items of interest could bring the viewer to the first-person perspective at the ground level. In addition, capabilities to filter out items that are not relevant to the task by applying dynamic queries in real time, including advanced filtering mechanisms to accommodate highly varied user needs, should be provided. Similarly the option to select an item or group and getting further details on demand would increase the usefulness of a visualization (Shneiderman, 1996). These principles, along with the somewhat similar "context plus focus" strategy (Baudisch et al., 2001), provide a framework for presenting and interacting with data and serve as foundation for the application concept in this project.

\subsection{Data Exploration in VR and Application Examples}

Data exploration in VR, specifically cultural heritage data by the means of interactive and immersive systems in particular appears to be still rare in practice. Many museums and collections do have an online presence, in which they offer search and filtering features to explore their inventory, or provide some interactive web maps and some use VR to create kind of a 'digital twin' of the museum. Using VR to present cultural heritage objects or phenomena, including historical journeys are found as physical exhibitions (Bruno et al., 2010, Yoo, Gold, 2019). Yet, seemingly the use of VR for data exploration is still largely uncharted in the cultural heritage domain. Nonetheless, there are some interesting examples. An impressive application in cultural heritage domain is the so-called mARChive, a stereoscopic interactive visualization environment that contains a collection of 100'000 objects from Melbourne's Museum Victoria. This navigable 360-degree data landscape offers an intuitive platform to engage with objects, which allows selection of items by topic and displays an image and meta data for selected data points on demand (Shaw et al., 2014).

There are also notable examples from related domains. For example, GeoSpace is one of the earliest interactive 3D visualization system that allows information seekers to explore complex information spaces. By putting strong emphasis on visual clarity, GeoSpace allows users to rapidly identify information in a dense display and it can guide a users' attention in a fluid manner while preserving overall context (Lokuge, Ishizaki, 1995). Similarly, a substantial fundamental work is presented with iViz, a tool for scientific data visualization, which has shown that immersion provides benefits beyond traditional visualization, and leads to a demonstrably better perception of datascapes, more intuitive data understanding and a better retention of perceived relationships in data (Donalek et al., 2014). And Open Data Exploration in Virtual Reality (ODxVR) proposes novel interface design approaches that enable embodied interaction (LaViola et al., 2017) and interactive visualization of open data within immersive VR environments. A particular focus of that project is placed on natural user interaction for information exploration (Reski, Alissandrakis, 2016). Last but not least, a recent landmark is the immersive VR visualization environment built for the ALSPAC data, which allows researchers to interact with and manipulate data doing remote collaborative analysis. It employs custom designed visualization methods that tap into the unique human ability to quickly recognize patterns in color, size, movement and spatial position. Researchers may explore data directly using freehand control to interact with data objects around them (Uni. Bristol, 2015).

The novelty of VaRt-DataExplorer lies in enabling spatial learning based on embodied interaction, immersive data visualization and exploration techniques with cultural heritage data collections, and designing this immersive data exploration environment to meet the special demands regarding visualization fidelity in this domain.

\section{MATERIALS AND METHODS}

\subsection{Visualization of Uncertainty}

A particular challenge working with cultural heritage data collections is dealing with incompleteness, imprecision and heterogeneity of data which is often only available in a semistructured format while many exploration techniques require harmonized datasets. Careful consideration must be given not to imply a higher level of detail (LoD) in the data transformation process than the original level of information (LoI). In cultural heritage reconstruction the difference between LoD and LoI is referred to as level of hypothesis (LoH) (Hauck, Kuroczynski, 2015). A specific example from our dataset is the geographical origin of collection items where only free text and no precise 
geolocation is provided. For many items only the country or a broader region within the country of origin is known. Placing an item marker such as a pin, flagpole or another kind of precise marker on a specific point on a map would be a hypothetical assumption whereas letting the objects float over a certain area is a better expression of the level of accuracy. Another example is the age of the artifact: For most objects, the dating does not yield a specific point in time but a vague textual description of a time interval (e.g., an estimated time span, a century or an epoch). Yet to dynamically process such information, it has to be converted into an interval of numeric values. As in the example above, careful consideration has to be given not to imply a higher LoI than what is actually available.

Uncertainty visualization, in general, is a difficult and persistent challenge (Çöltekin et al., 2017). Many scholars stress the importance of a truthful representation of incompleteness and uncertainty (MacEachren et al., 2005). In the context of cultural heritage, and specifically as a remedy for the mismatch between the LoI and LOD, it is recommended that one purposefully devises visual representation forms with 'adequate or lower' LoD to demonstrate the problem (Gershon, 1998). Another study recommends to present data together with auxiliary uncertainty information because these visualizations present a more complete and accurate rendition of data for users to analyse (Pang et al., 1997). Somewhat promising at first, archival databases typically consist of (pictures of) artifacts, and metadata representing the actual object. Yet this documentation layer is merely an image of the original, and it should be taken into account that the reproduction might not be complete, nor fully representing it substantiality (Koebel et al., 2017). In sum, designing display modes for $2 \mathrm{D}$ artifacts in a $3 \mathrm{D}$ environment have to be carefully considered not to mislead viewers.

\subsection{Representing the World in VR}

Since we focus on geographically referenced global data, one of the challenges is to represent the map of the world in VR. To represent the world in VR, typically globes are used. A globe can be exocentric (viewpoint outside the globe) or egocentric (viewpoint inside the globe); and one can transition between these two views. To use the traditional cartographic maps in $\mathrm{VR}$, one needs to re-project them to curved surfaces such as on a portion of a sphere (Yang et al., 2018). The question remains whether virtual globes are the best way to display global data in immersive environments, or if another representation type might be more useful in some cases (Çöltekin et al., 2019b). A significant limitation of globes is that only one hemisphere is visible at once, and some users may be unfamiliar with this perspective, which may lead to spatial disorientation. Given these concerns and the Shneiderman's "overview first" principle; to provide global spatial context, we decided to present the data on a traditional cartographic map (thus projected on a flat plane).

\subsection{System Control}

After a qualitative evaluation of the options (i.e., reading and brainstorming sessions with the research team), we decided that 'body referenced controls' (LaViola et al., 2017) - sometimes also called 'control in hand' (Sherman, Craig, 2018) -, attached to the hand controllers might be best solution for this use case as they minimize occlusion, yet remain available to the user anytime, and are therefore easy to utilize. For menu design, user studies comparing pie menus to linear menus have shown that radial placement reduces target selection time and lowers error rate (Ryan et al., 2008), thus we opted for this solution. Regarding spatial movement, a combination of physical (real) walking movement and teleportation was chosen. While the first allows continuous motion, it's interaction space is limited to the size of the game space (Boletsis, 2017). The second artificial interaction type supplements this limitation. Teleportation is one of the most prevalent and easy to learn locomotion techniques for virtual spaces. In addition, this technique is frequently used since it is less tiring and less prone to cause motion sickness than other locomotion techniques, yet a downside is that the sudden jumps may cause disorientation (Bond, Nyblom, 2019, Coomer et al., 2018).

\subsection{User Experience and Usability Testing}

Throughout the project's life cycle, we held a number of qualitative sessions with potential users which informed interaction and visualization related implementation decisions. Once the prototype was ready, we conducted a final test for usability and additional user feedback. This final test was mainly to understand the usability of the system, and for this purpose a participant sample of 6 is considered sufficient (Nielsen, 2012). We also asked additional questions to gauge pragmatic and hedonistic qualities such as perceived ease of use of specific features, comprehensibility of data visualization, performance, enjoyability and insights gained to obtain early indicators on these topics. While usability findings should be conclusive, we consider the other observations similar to a pilot study, i.e., these findings will inform a future controlled lab study.

During the procedure of the experiment, the context and overarching idea were briefly explained and features of the application were demonstrated to the participants prior to letting themselves explore the virtual data space and 'think aloud' (Eccles, Arsal, 2017). Then, they were given specific tasks to solve. Specifically, they were asked to change the perspective, retrieve detailed information about a collection item and apply filters to the dataset. At the end they were also asked to share their interpretation (qualitative opinions) of the visualization concept verbally. Following the test, we administered a battery of questions we prepared on participants' experience on the usability of specific features of the application, how they estimate their own performance in the given test tasks as well as their overall user experience. We have also asked participants to rate their experience in terms of 'enjoyablity' and 'insight gained'. All rating questions were asked using a 5-point Likert scale.

\section{RESULTS}

The key outcome from this project is a prototype implementation, and the user reactions to it. A video illustration of the prototype implementation can be seen at https://vimeo.com/ 328613493 .

\subsection{Layers of Visualization and Dimensions of Interaction}

Our data visualization concept aims to combine visualization of the dataset as a whole with detailed information about specific data points, and therefore operates on two layers (see Figure 1): The former is incorporated into the visualization in the form of a choropleth map (color coding plus encoding volume height adjustments, see Figure 2), and the latter is represented in the display of the individual collection objects (see Figure 4).

Developing the interaction concepts followed similarly complex considerations. The exploration space is 'polyvalent' i.e., 


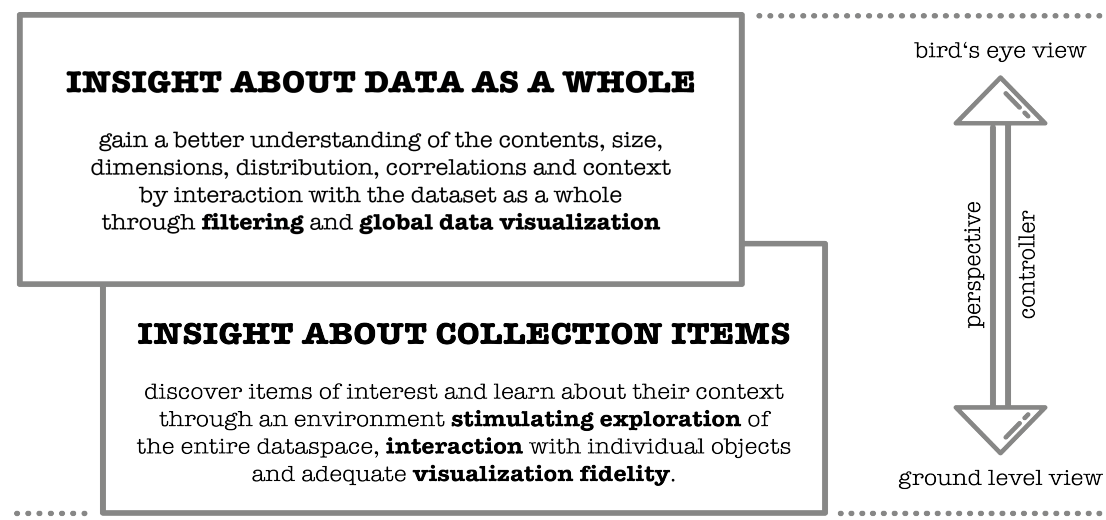

Figure 1. Two layers of the visualization concept: higher level for global data visualization and lower level to display information on individual collection items.

it offers interaction possibilities with different degrees of abstraction and focus, both with the dataset as a whole, and with individual collection items. Firstly, on a rather abstract level, interaction with the entire dataset may be performed by applying filters (see Figure 3) which should lead to a better understanding of distribution, correlations and potentially the discovery of patterns. Secondly, still indirect, but slightly more tangible interaction with data is possible by pointing at individual data points in order to obtain details on demand (see Figure 4). And last but not least, users may experience embodied interaction by approaching collection objects themselves and obtain a more comprehensive appreciation of individual items, accompanied by insight on their geographical context and surroundings.

\subsection{Considerations regarding Representation Fidelity}

Besides visualization and interaction design considerations, we have taken into account uncertainty and devised concepts in consideration to the LoD of the data points. Mainly, where we could not georeference the data to its precise origin (as mentioned in section 3.1, the information provided in the collection database is rather vague for some objects and for others, the geographic information about the place of origin is ambiguous), placing a collection item on a specific spot would have implied a higher LoI than data actually allows. Therefore we did not place the collection items directly on the globe, but let them float slightly over the estimated region which we decided was a better expression of the given level of accuracy. For the same reason, the choropleth map was only applied on country level.

\subsection{Features of the Functional Prototype}

A key outcome from this research so far is a functional prototype implemented with Unity (Unity, 2020) using the VRTK framework (Fox, Extend Reality Ltd, 2018). The current prototype is optimized for the HTC Vive (HTC Corporation, 2020) headset and controllers. As shown in Figure 2, the prototype contains a map that provides an overview of the full dataset first. This view is further supported by a body referenced on-demand menu for filtering data points by various criteria such as region of origin, time period, material or presence in exhibition (see Figure 3), and visualization concepts for direct integration of filter results in the map representation. This immediate spatial representation of search results offers rapid insight on the contents of the dataset to the user. Applying Shneiderman's "details on demand" principle, a detail view with additional information is displayed by pointing at collection items. Exemplary samples for the representation of objects with respect to their LoD have also been implemented (see Figure 4). The application provides system control by the means of a perspective controller, allowing zooming in and out to shift the user's perspective back and forth between bird's eye view and ground level view (see Figure 3). Spatial movement on the map is offered by physical locomotion and teleportation.

\subsection{Data and Application Management}

Data management is provided through a JSON interface (ECMA, 2017), which enables automated import of data into the application by the means of JSON files containing the collection objects' meta information. Rietberg uses the museum collection management software 'MuseumPlus' (zetcom, 2020) for maintaining the data. Yet the chosen approach allows data to be imported from any type of database. Also the overall design of the application management is modular: at the launch of the experience cultural heritage objects are generated dynamically, combing a photograph of the object with meta information extracted from the corresponding entry in this JSON file. Data entries contain the approximated latitude and longitude coordinates which are translated into $\mathrm{x}$ and $\mathrm{y}$ values in respect to the Mercator projection used in the map representation, and objects are then placed above the approximate geographic location (see considerations in 4.2 ).

As the application targets a non-expert general audience of art enthusiasts and parties with strong interest in cultural heritage who care much about the visual representation, the application including the management of LoDs is custom built in Unity without any underlying geographic information system (GIS). This allows full control over the visualization style and the resulting user experience of the application in general, as well as the proposed immersive data analytics concept in particular. Each country (on the map representation) is an individual 'object' made with Blender (Blender, 2020), whose height and texture color are adjusted depending on the number of items currently selected in this particular region to create the subjacent choropleth map. And depending on the user's current view, the corresponding LoD of the objects is loaded.

\subsection{Results from User Experience and Usability Testing}

The prototype was tested with 8 participants (ages 22-35, 6 men, 2 women) with diverse occupational backgrounds. 7 out of 8 had little experience with VR, stating that they had tried it once, twice or 'only a few times' before. Yet, half of the 


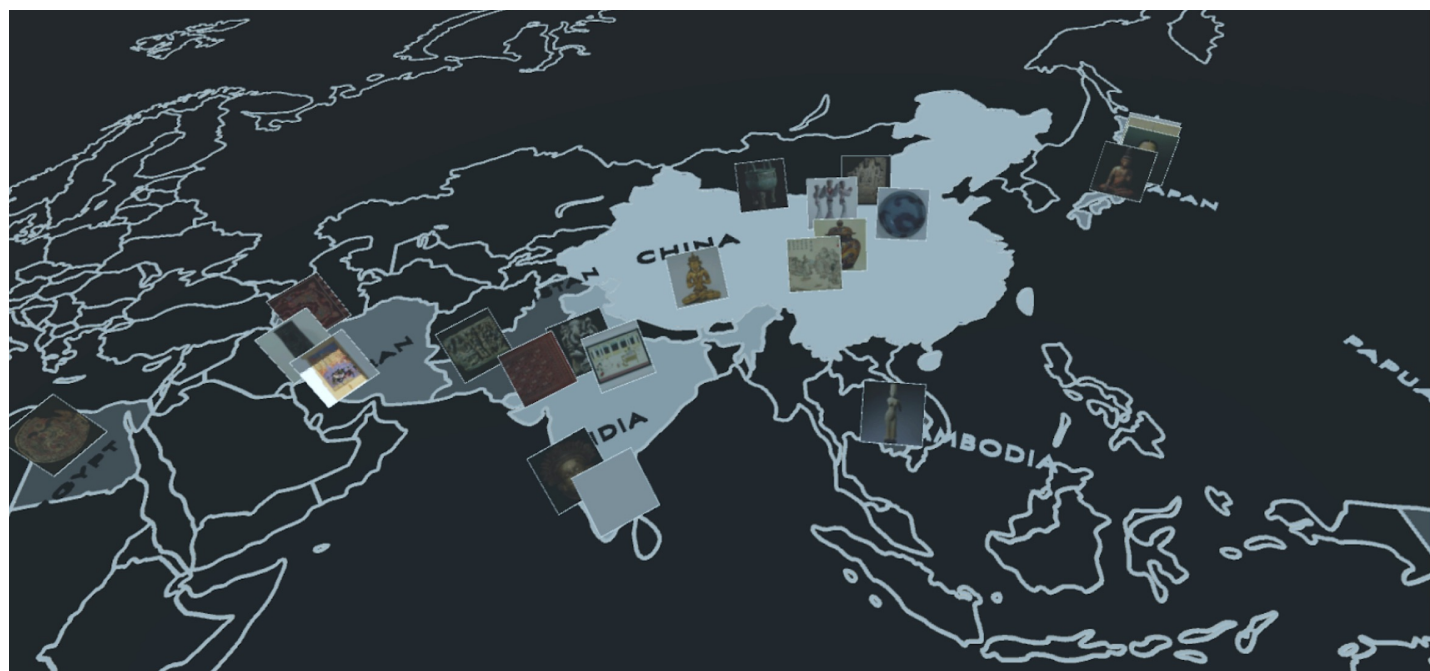

Figure 2. Overview of the entire collection (bird's eye view perspective) following the "overview first" principle by Shneiderman to let the user gain an overview of the contents of the entire collection. On the subjacent choropleth map layer, countries with large amounts of collection items matching the current filter settings are highlighted. Darker colors mean less instances in the collection.

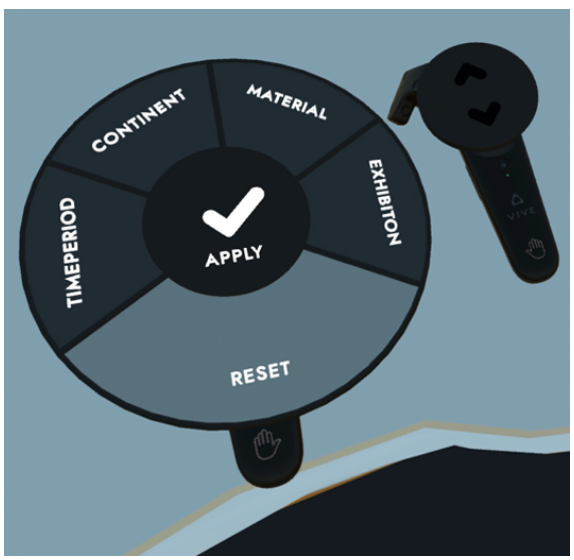

Figure 3. Radial filter menu to refine selection and perspective controller to shift the user's perspective back and forth between bird's eye view and ground level view.

participants were experienced with computer games, i.e., marking their experience as 4 or 5 on a 5-point Likert scale. Concerning usability, 5 out of 8 participants rated the application overall 'easy' or 'rather easy' to use and 2 other gave a rating of 3 on a 5-point Likert scale. For the specific tasks the results varied slightly: Locomotion in the virtual space, application of filters and retrieval of detail information about collection items was intuitive to 6 out of 8 participants. The operation of the perspective controller was a little bit more challenging for some of the participants, it was rated as intuitive only by half of the participants, another 3 reported it as somewhat intuitive. The choropleth map was only comprehensible for half of the participants on first sight, yet after explaining the feature, its meaning became obvious to the remaining users. Regarding performance, 6 participants considered that they completed the given tasks successfully whereas 2 reported that they were unsure about their achievements. Importantly, 7 out of 8 considered the experience to be 'very enjoyable' or 'enjoyable', and all reported that the application offered them a fresh perspective on the dataset ( 7 reported the maximum number of points, one marked 4/5). However, insights gained by the exper-

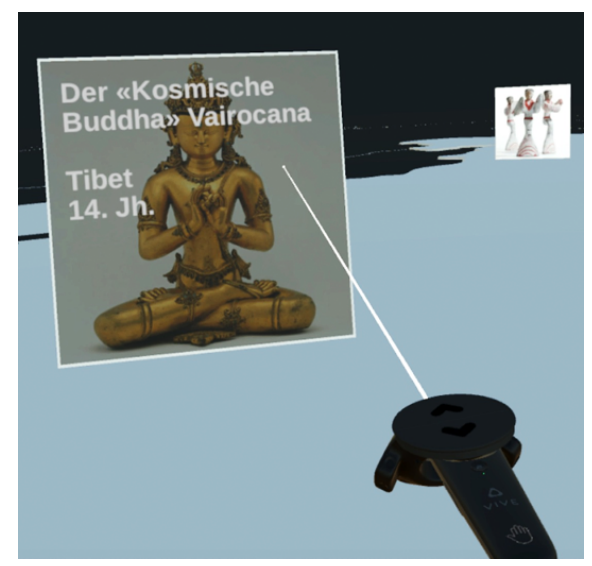

Figure 4. Detail view (ground level perspective): more information (e.g., object title, place and time of origin) may be requested on demand by pointing at a collection item.

ience were perceived slightly less uniformly: Half reported that they gained 'much' or 'rather much' new insight whereas the other half declared that 'some', 'rather little' or 'little' insight was gained.

\section{DISCUSSION AND CONCLUSION}

\subsection{Meaning and Measuring of Insights Gained}

Regarding insights gained the application, there was a higher perception of new insight gained by the more experienced participants. This might not be by coincidence but rather because this cohort learned quicker how to operate and navigate in the virtual world, and as a consequence were able to focus more on content rather than system control. Another explanation for the somewhat mixed feedback on new insights could be varying levels of previous knowledge of the application domain and different expectations or personal definition of what is considered much or little new insight. To obtain more reliable results on this subject, standardised scales for measuring 
user experience and user engagement, e.g., User Experience Questionnaire (UEQ, 2018) or User Engagement Scale (UESSF) (O'Brien et al., 2018), should be used and user behaviour should be measured in addition to self reported feedback.

\subsection{D Representation of the Collection Items}

Some observations regarding the technical challenges as well as user experience with a VR data space in the context of cultural heritage, that may be of use to others who may want to explore a similar idea, include the starting data: Even though the collection mainly consists of physical objects, the database contains no 3D models (only 2D photographs plus metadata for the objects). For this prototype, we did not attempt creating 3D models of the objects based on the data provided. This would have been possible if one takes some artistic liberty (Hassner, Basri, 2006), but if precision is important, these can be created e.g., by the means of photogrammetric scans (Bruno et al., 2010), though it would be a delicate procedure given the fragile substance of some of the historical objects. Yet if 3D models of the sculptures eventually are integrated in a prototype like ours, we believe an immersive experience will be of even greater value to users, as VR would enable exploring the sculptures from any perspective in their real world size. Based on our previous own work, we believe that not only the user experience and gained insights, but also the memorability (information retention and recall) would improve by using a well-designed virtual representation (Lokka, Çöltekin, 2019), which would be of relevance from an educational perspective. A natural future step is to create $3 \mathrm{D}$ models for a subset of the collection and measure the memorability of that alternative against the current experience.

\subsection{Visualization Design Decisions}

It is evident that there is no neutral way to visually represent data points, each form of visualization carries its own underlying message. There are many factors to consider when making choices about the visual representation style starting with well-known visual variables such as color, shape, style, perspective, size and amount of data on display, medium, context, etc. (Bertin, 1983, Rautenbach et al., 2015). Yet there are also many more less obvious design decisions that need to be considered. For example, visualizing temporal evolution as the objects in the collection cover a long period of time over which many geopolitical changes have occurred is an interesting challenge. Some collection items contain descriptions of historical geographical locations such as 'Maya lowland' for example. How can the change of political structures over time be represented appropriately and integrated in such a VR experience? The challenge posed regarding the visualization of the geographical context is to find the right balance between historical fidelity on one side of the spectrum and orientation, comprehensibility and usability on the other side. An additional challenge to keep in mind related to the map representation, is finding a sensible approach to political issues such as disputed country borders and autonomous or independent regions.

\subsection{Orientation and Navigation in the Virtual Space}

While striving to offer the highest degree of freedom to the users to explore the virtual (data) space at their own discretion, it is important to remember that we, as designers of such systems, need to offer some support for orientation and navigation for the users to take advantage of this freedom. Without a wellconsidered interaction design, the exploration process will not succeed. We noticed that as long as the user observes the data space from the bird's eye perspective, orientation seems obvious in spite of minimal features given (country boarders only) thanks to the familiarity of this abstract map representation. Yet we further observed that a real challenge is to keep orientation when users switch perspective to ground level view. Adding additional geographical features such as rivers, mountain ranges or other landmarks might offer additional orientation support. Another common idea to solve this problem is offering details about collection items by the means of magnification of the selected item from bird's view perspective instead of having to approach it for this purpose. However, we hold the view that the spatial movement intensifies the exploration process.

\subsection{Onboarding Strategies for Users with no or little VR Experience}

Most members of the envisaged target group (non-expert users) are assumed to be inexperienced with VR technology. Based on our observations among the participants with little or no VR experience, we noticed the need of clear instructions about the use of the hardware, the controllers in particular. Explanations or a brief training session on how to navigate in the virtual space and how to make use of the features provided by the application are important to take full advantage of the proposed data exploration experience. This could be done, for example, by means of an interactive tutorial where the user learns the functionality of all the features offered. In addition, it can be useful to guide the user through a specific use case to become familiar with the application. A potential future implementation could demonstrate usage and interaction principles by applying a narrative pattern called Martini glass structure (Segel, Heer, 2010) to bring users all at the same level of familiarity with the case. This 'Martini glass structure' begins with a single-path author driven approach following a tight narrative path representing the stem of the glass. Once the intended narrative is complete, the experience opens up to a user-driven exploratory stage where the user is free to interactively explore the data representing the body of the glass (Segel, Heer, 2010).

\subsection{Transferability of Findings}

Recognizing that visuospatial representation, filtering and interaction capabilities provided by the VaRt-DataExplorer result in a fresh and unique perspective, which brings about new insight on data and increased joy of use by the target audience, applying these findings to other georeferenced datasets might be interesting to consider. In fact, many museums and archives have large amounts of reusable yet largely unexploited documentation data, and this latent potential is not limited to the domain of cultural heritage alone. It can be unlocked if data is presented to the public in a meaningful way and made accessible for interaction, exploration and discovery. And the outcome generated through processing this documentation layer may eventually become an actual experience of its own. For this reason, every endeavour should be made towards reusability. This implies in particular advancements in the data management interface and modularity of the application architecture as well as on the elaboration of concepts to embed such a VR experience into the context of an online presence or as part of an exhibition.

\subsection{Future Work}

Initial user tests have resulted in an overall positive feedback, suggesting that this form of spatial visualization and interaction with data not only have the potential to provide tangible 
benefits for data exploration, but also contains hedonistic qualities. These hedonistic qualities should not be underestimated, when people enjoy a system or a visualization, they are more likely to re-use the system and retain the information. As a next step, more systematic testing is necessary to confirm these initial observations, such as controlled experiments to test the hypotheses we have developed based on this study.

Regarding data visualization, further elaboration on expedient visual variables for embedding search query results into the map representation are necessary. 3D is not always 'best' for everything. For example, scale variation in 3D makes judging distances, heights and areas harder, and at times occlusion removes relevant information from the field of view (Çöltekin et al., 2016). A combination of spatial data representation together with a visual encoding in the texture of the surface of a geographic object might facilitate a faster interpretation of data. Other future work includes refinement of menu design and introduction of more elaborated search and filtering options (e.g., allowing the combination of multiple criteria), yet the default filter setting should be a combination that provides an interesting selection of items. For the detail view the display of additional attributes or behaviors of objects such as its provenance journey, plus interactions like marking favorites or comparing the behavior of multiple selected objects are envisioned. Furthermore, freehand system control should be considered in a future implementation. The benefit of gesture input lies in the natural characteristics of direct mapping of user movement which are easier to learn than virtual control performed by an input device. Studies have shown that bimanual input in particular has the potential to allow users to perform tasks faster, leverage existing skills, and increase expressiveness (Owen et al., 2005). A drawback is the need for clear delimiters to indicate initialization and determination of gestures, otherwise unintentional human motions may be interpreted as gestures.

With further refinement of the visualization and interaction design (possibly in the direction we speculated above, and in parts of the Discussion section), and a series of controlled lab experiments, our observations could become more robust; and our implementation, as well as others that have similar goals to visualize cultural heritage data in VR, would be more usable and more useful. Nonetheless, these initial prototype and observations from the user experience study gives us valuable insights towards further research.

\section{REFERENCES}

Baudisch, P., Good, N., Stewart, P., 2001. Focus plus context screens: combining display technology with visualization techniques. Proceedings of the 14th Annual ACM Symposium on User Interface Software and Technology, 31-40.

Bertin, J., 1983. Semiology of Graphics: Diagrams. Networks, Maps, 10.

Blackler, A., Popovic, V., Mahar, D., 2010. Investigating users' intuitive interaction with complex artefacts. Applied Ergonomics, 41(1), 72-92.

Blender, 2020. Open source 3D creation. https://www. blender.org (last accessed 02/05/2020).

Boletsis, C., 2017. The new era of virtual reality locomotion: a systematic literature review of techniques and a proposed typology. Multimodal Technologies and Interaction, 1(4), 24.
Bond, D., Nyblom, M., 2019. Evaluation of four different virtual locomotion techniques in an interactive environment. Blekinge Institute of Technology, Karlskrona, Sweden.

Bruno, F., Bruno, S., De Sensi, G., Luchi, M.-L., Mancuso, S., Muzzupappa, M., 2010. From 3D reconstruction to Virtual Reality: A complete methodology for digital archaeological exhibition. Journal of Cultural Heritage, 11(1), 42-49.

Chandler, T., Cordeil, M., Czauderna, T., Dwyer, T., Glowacki, J., Goncu, C., Klapperstueck, M., Klein, K., Marriott, K., Schreiber, F. et al., 2015. Immersive analytics. IEEE 2015 Big Data Visual Analytics (BDVA), 1-8.

Cliquet, G., Perreira, M., Picarougne, F., Prié, Y., Vigier, T., 2017. Towards HMD-based immersive analytics. Immersive analytics Workshop, IEEE VIS 2017, Phoenix, USA.

Çöltekin, A., Bleisch, S., Andrienko, G., Dykes, J., 2017. Persistent challenges in geovisualization-a community perspective. Int. Journal of Cartography, 3(1), 115-139.

Çöltekin, A., Christophe, S., Robinson, A., Demšar, U., 2019a. Designing Geovisual Analytics Environments and Displays with Humans in Mind. ISPRS Int. J. Geo-Inf., 8(12), 1-6.

Çöltekin, A., Lokka, I.-E., Zahner, M., 2016. On the usability and usefulness of 3D (geo) visualizations - A focus on virtual reality environments. Int. Archives of the Photogrammetry, Remote Sensing \& Spatial Information Sciences, XLI-B2, 387392.

Çöltekin, A., Oprean, D., Wallgrün, J. O., Klippel, A., 2019b. Where are we now? Re-visiting the Digital Earth through human-centered virtual and augmented reality geovisualization environments (Editorial). Int. Journal of Digital Earth, 12(2).

Coomer, N., Bullard, S., Clinton, W., Williams-Sanders, B., 2018. Evaluating the effects of four VR locomotion methods: joystick, arm-cycling, point-tugging, and teleporting. Proceedings of the 15th ACM Symposium on Applied Perception, 1-8.

Donalek, C., Djorgovski, S. G., Cioc, A., Wang, A., Zhang, J., Lawler, E., Yeh, S., Mahabal, A., Graham, M., Drake, A., Davidoff, S., Norris, J., Longo, G., 2014. Immersive and collaborative data visualization using virtual reality platforms. 2014 IEEE Int. Conference on Big Data, IEEE, 609-614.

Eccles, D. W., Arsal, G., 2017. The think aloud method: What is it and how do I use it? Qualitative Research in Sport, Exercise and Health, 9(4), 514-531.

ECMA, 2017. The JSON Data Interchange Syntax, 2nd Edition. https://www.ecma-international.org/publications/files/ECMAST/ECMA-404.pdf (last accessed 05/04/2020).

Fox, T. E., Extend Reality Ltd, 2018. Virtual Reality Toolkit. https://www.vrtk.io (last accessed 05/03/2020).

Gershon, N., 1998. Visualization of an imperfect world. IEEE Computer Graphics and Applications, 18(4), 43-45.

Hassner, T., Basri, R., 2006. Example based 3D reconstruction from single 2D images. 2006 Conference on Computer Vision and Pattern Recognition Workshop (CVPRW'06), IEEE, 15-15.

Hauck, O., Kuroczynski, P., 2015. Cultural Heritage Markup Language: How to record and preserve 3D assets of digital reconstruction. CHNT 20: 20th Int. Conference on Cultural Heritage and New Technologies 2015. 
HTC Corporation, 2020. What is high-end Virtual Reality? https://www.vive.com/us/comparison (last accessed 05/03/2020).

Hutchins, E. L., Hollan, J. D., Norman, D. A., 1985. Direct manipulation interfaces. Human-Computer Interaction, 1(4), 311338.

Idreos, S., Papaemmanouil, O., Chaudhuri, S., 2015. Overview of data exploration techniques. Proceedings of the 2015 ACM SIGMOD Int. Conference on Management of Data, 277-281.

Koebel, K., Agotai, D., Arisona, S., Oberli, M., 2017. Biennale $4 \mathrm{D}$ - a journey in time: Virtual reality experience to explore the archives of the Swiss pavilion at the "Biennale di Venezia" art exhibition. 2017 23rd Int. Conference on Virtual System \& Multimedia (VSMM), IEEE, 1-8.

LaViola, J. J., Forsberg, A. S., Laidlaw, D. H., Van Dam, A. et al., 2009. Virtual reality-based interactive scientific visualization environments. Trends in Interactive Visualization, Springer, 225-250.

LaViola, J. J., Kruijff, E., McMahan, R. P., Bowman, D., Poupyrev, I. P., 2017. 3D User Interfaces: Theory and Practice. Addison-Wesley Professional.

Lokka, I. E., Çöltekin, A., 2019. Toward optimizing the design of virtual environments for route learning: empirically assessing the effects of changing levels of realism on memory. Int. Journal of Digital Earth, 12(2), 137-155.

Lokuge, I., Ishizaki, S., 1995. Geospace: An interactive visualization system for exploring complex information spaces. Proceedings of the SIGCHI conference on Human factors in computing systems, 409-414.

MacEachren, A. M., Robinson, A., Hopper, S., Gardner, S., Murray, R., Gahegan, M., Hetzler, E., 2005. Visualizing geospatial information uncertainty: What we know and what we need to know. Cartography and Geographic Information Science, 32(3), 139-160.

McIntire, J. P., Havig, P. R., Geiselman, E. E., 2014. Stereoscopic 3D displays and human performance: A comprehensive review. Displays, 35(1), 18-26.

Museum Rietberg, 2020. Sammlung Online. http://www.samm lungrietberg.ch/eMuseumPlus (last accessed 05/03/2020).

Napier, J., Napier, J. R., Tuttle, R. H., 1993. Hands. 9, Princeton University Press.

Nielsen, J., 2012. How many test users in a usability study. Nielsen Norman Group, 4(06).

Owen, R., Kurtenbach, G., Fitzmaurice, G., Baudel, T., Buxton, B., 2005. When it gets more difficult, use both hands: exploring bimanual curve manipulation. Proceedings of Graphics Interface 2005, Canadian Human-Computer Communications Society, 17-24.

O’Brien, H. L., Cairns, P., Hall, M., 2018. A practical approach to measuring user engagement with the refined user engagement scale (UES) and new UES short form. Int. Journal of Human-Computer Studies, 112, 28-39.

Pang, A. T., Wittenbrink, C. M., Lodha, S. K. et al., 1997. Approaches to uncertainty visualization. The Visual Computer, 13(8), 370-390.
Rautenbach, V., Coetzee, S., Schiewe, J., Çöltekin, A., 2015. An assessment of visual variables for the cartographic design of 3D informal settlement models. Proceedings of the 27th Int. Cartographic Conference, August 23-28, Rio de Janeiro, ICA, Int. Cartographic Association, ISBN 978-85-88783-11-9.

Reski, N., Alissandrakis, A., 2016. Change your perspective: exploration of a 3D network created from open data in an immersive virtual reality environment. ACHI 2016: The 9th Int. Conference on Advances in Computer-Human Interactions, IARIA, 403-410.

Ryan, T. N., McMahan, P., Bowman, D. A., 2008. Tech-note: rapmenu: remote menu selection using freehand gestural input. 2008 IEEE Symposium on 3D User Interfaces, IEEE, 55-58.

Schnürer, R., Ritzi, M., Çöltekin, A., Sieber, R., 2020. An empirical evaluation of three-dimensional pie charts with individually extruded sectors in a geovisualization context. Information Visualization, 1473871619896103.

Segel, E., Heer, J., 2010. Narrative visualization: Telling stories with data. IEEE Transactions on Visualization and Computer Graphics, 16(6), 1139-1148.

Shaw, J., Kenderdine, S., Hart, T., 2014. mARChive. Centre for Interactive Cinema Research. http://www.icinema. unsw.edu.au/projects/marchive/project-overview (last accessed 05/04/2020).

Shepherd, I. D., 2008. Travails in the third dimension: a critical evaluation of three-dimensional geographical visualization. Geographic visualization: Concepts, tools and applications (Eds. M. Dodge et al.), John Wiley \& Sons, 199-222.

Sherman, W. R., Craig, A. B., 2018. Understanding Virtual Reality: Interface, Application and Design. Morgan Kaufmann.

Shneiderman, B., 1996. The eyes have it: A task by data type taxonomy for information visualizations. Proceedings 1996 IEEE symposium on visual languages, IEEE, 336-343.

Stephanidis, C., Salvendy, G., Antona, M., Chen, J. Y., Dong, J., Duffy, V. G., Fang, X., Fidopiastis, C., Fragomeni, G., Fu, L. P. et al., 2019. Seven HCI grand challenges. Int. Journal of Human-Computer Interaction, 35(14), 1229-1269.

UEQ, 2018. User Experience Questionnaire. https://www.ueqonline.org (last accessed 05/04/2020).

Uni. Bristol, 2015. Virtual Reality helps make sense of complex scientific data. http://www.bristol.ac.uk/alspac/news/2015/vrbig-data-prize.html (last accessed 05/03/2020).

Unity, 2020. Unity for all. https://unity.com (last accessed 05/03/2020).

Wagner Filho, J. A., Rey, M. F., Freitas, C. M., Nedel, L., 2018. Immersive visualization of abstract information: An evaluation on dimensionally-reduced data scatterplots. 2018 IEEE Conference on VR and 3D User Interfaces (VR), IEEE, 483-490.

Yang, Y., Jenny, B., Dwyer, T., Marriott, K., Chen, H., Cordeil, M., 2018. Maps and globes in virtual reality. Proceedings of Eurographics Conference on Visualization (EuroVis), Computer Graphics Forum, 37, Wiley Online Library, 427-438.

Yoo, K., Gold, N., 2019. Emotion evoking art exhibition in VR. 25th ACM Symposium on VR Software and Technology, 1-2.

zetcom, 2020. MuseumPlus. https://www.zetcom.com/museum plus (last accessed 05/03/2020). 